The ASTRONOMICAL JOURNAL, 119:1534-1541, 2000 April

(C) 2000. The American Astronomical Society. All rights reserved. Printed in U.S.A.

\title{
THE INCIDENCE OF THE HOST GALAXY IN MICROVARIABILITY OBSERVATIONS OF QUASARS
}

\author{
S. A. CELLONE ${ }^{1}$ \\ Facultad de Ciencias Astronómicas y Geofísicas, Universidad Nacional de La Plata, Paseo del Bosque, 1900 La Plata, Argentina; \\ scellone@fcaglp.unlp.edu.ar \\ AND \\ G. E. ROMERO ${ }^{1,2}$ AND J. A. COMBI ${ }^{1,2}$ \\ Instituto Argentino de Radioastronomía, C.C. 5, 1894 Villa Elisa, Argentina; romero@irma.iar.unlp.edu.ar, combi@irma.iar.unlp.edu.ar \\ Received 1999 August 30; accepted 1999 December 15
}

\begin{abstract}
The incidence of a host galaxy in aperture photometry of active galactic nuclei is studied by means of actual and simulated CCD observations. Our goal is to evaluate the importance of spurious variations, introduced by seeing fluctuations during the observations, in the differential light curves used to study optical microvariability. Repeated CCD observations during two consecutive nights were used to obtain time-resolved aperture photometry for the BL Lac object PKS 2316-423, which is located at the center of a conspicuous elliptical galaxy, and for several field stars. The blazar seems to be variable according to standard variability criteria; however we show that the observed differential magnitude variations are strongly correlated with seeing fluctuations during the nights. Moreover, another galaxy within the same CCD field shows nearly identical variations, clearly indicating that such variations are artifacts of the aperture photometry. Simulated observations of quasars within host galaxies of different morphologies and spanning a wide range of luminosities were also used to evaluate the effects of changing seeing conditions. The results show that spurious differential magnitude variations due to seeing fluctuations are larger for active nuclei within brighter hosts, particularly when small photometric apertures (about the seeing FWHM in radius) are used. According to our results, several recommendations are given to future observers.
\end{abstract}

Key words: galaxies: photometry — quasars: individual (PKS 2316-423)

\section{INTRODUCTION}

CCD-based differential photometric observations of rapid variability over timescales of significantly less than a day in the optical emission of active galactic nuclei (AGNs) are a powerful tool for probing the innermost regions of these objects. The existence of such luminosity changes, usually known as "microvariability," was established in the late 1980s and early 1990s by means of detailed observations made by Miller, Carini, \& Goodrich (1989), Carini, Miller, \& Goodrich (1990), Carini et al. (1991, 1992), and others. Most radio-loud quasars and blazars appear to display high duty cycles of microvariability with amplitudes up to several tenths of a magnitude within a single night (e.g., Miller \& Noble 1996 and references therein). Radioquiet quasars seem to present a lower level of activity (e.g., Gopal-Krishna, Sagar, \& Wiita 1993a; Gopal-Krishna, Wiita, \& Altieri 1993b; Gopal-Krishna, Sagar, \& Wiita 1995; Sagar, Gopal-Krishna, \& Wiita 1996; Rabette et al. 1998).

The origin of the microvariability phenomenon is still unknown. Models involving shock propagation through relativistic jets (e.g., Marscher, Gear, \& Travis 1992) and accretion disk instabilities or perturbations (e.g., Mangalam \& Wiita 1993; Chakrabarti \& Wiita 1993) are usually invoked. Large-sample monitoring programs of both radioloud and radio-quiet objects could contribute with valuable

\footnotetext{
${ }^{1}$ Visiting Astronomer, Complejo Astronómico El Leoncito, operated under agreement between the Consejo Nacional de Investigaciones Científicas y Técnicas de la República Argentina and the Universities of $\mathrm{La}$ Plata, Córdoba, and San Juan.

${ }^{2}$ Member of CONICET.
}

information to solve the problem of where and how the microvariations are produced (e.g., Jang \& Miller 1995, 1997; Romero, Celone, \& Combi 1999).

The basic technique used in microvariability observations of AGNs is CCD $N$-star photometry (e.g., Howell \& Jacoby 1986; Carini et al. 1991). Stars in the same frame containing the AGN are used for multiple checking. Ideally, stellar light curves can be used to remove spurious variations introduced by fluctuations in the atmospheric transparency or extinction. The results are generally presented as differential light curves $O-C_{i}$, where $O$ is the observed magnitude of the object under scrutiny and $C_{i}$ is the magnitude of a comparison star. Additional $C_{i}-C_{j}$ curves are used to estimate the observational errors.

A possible source of error in observations of this kind is the presence of an AGN host galaxy. The surface brightness profile of any galaxy will generally not behave like a point source does when convolved with the seeing point-spread function (PSF); thus intranight fluctuations in the seeing could result in a variable light contribution through the photometric aperture, producing spurious changes in brightness that could be mistaken for AGN microvariability. This problem has been discussed by Carini et al. (1991) in connection with their study of AP Librae. These authors have tested the influence of an extended underlying galaxy through observations of the active nucleus of the Seyfert galaxy MCG 8-11-11. They showed that in this particular case major extrinsic fluctuations in the light curves are removed in the process of differential photometry, and they concluded that "any spurious variations that are the result of seeing and/or transparency changes are small compared to the other sources of error considered and certainly could not be responsible for the linear trends observed for 
AP Librae." The reader is referred to Carini et al.'s paper for further details.

Since Carini et al. (1991) conducted their study on MCG 8-11-11, most authors just quote their conclusions and do not take any further precautions against the problem of the possible galaxy contribution to microvariability observations. The aim of the present paper is to show that, in some cases, such a confident attitude is not justified and to provide some quantitative elements to optimize the results of microvariability monitoring of AGNs. We think, in the light of some contradictory claims in microvariability findings, that this is a worthy task.

We have observed a BL Lac object (PKS 2316-423) with a significant galactic component and located in a cluster of galaxies, in such a way that, for variability control, we could have different extended objects available within the same CCD frame in addition to several stars. With these observations, we proved that spurious effects are present when seeing conditions fluctuate and that they cannot be completely removed by differential photometry with stars alone. We have then made model simulations of varying observing conditions in order to weight the contributions of the different parameters involved in the observations.

The structure of the paper is as follows. In $\S 2$ we provide information on the studied objects and the observations. In $\S 3$ we give the results of these observations and demonstrate the reality of the spurious contributions. Section 4 deals with model simulations of the underlying galactic contribution to microvariability. In $\S 5$ we discuss the results, and, finally, in $\S 6$ we draw our conclusions.

\section{OBSER VATIONS}

PKS 2316-423 is classified as an X-ray selected BL Lac object by Padovani \& Giommi (1995). At redshift $z=0.055$, it is associated with the brightest member of the Sersic 159-02 (S1111) cluster of galaxies. The AGN elliptical host galaxy was studied by Crawford \& Fabian (1994), who reported results of optical, ultraviolet, and X-ray observations, revealing a continuum spectrum with strong nonthermal contribution from the central source.

We have observed PKS 2316-423 during two consecutive sessions on 1997 September 4 and 5 with the CASLEO $2.15 \mathrm{~m}$ telescope located at San Juan, Argentina. The nights were nonphotometric, with extinction and seeing conditions fluctuating because of thin cirrus. The instrument was equipped with a liquid-nitrogen-cooled CCD camera using a Tek 1024 chip, with a read-out noise of 9.6 electrons and a gain of 1.98 electrons $\mathrm{adu}^{-1}$. The scale was 0.82 pixel $^{-1}$, and the field of view was about 700 pixels, thus corresponding to $9^{\prime}$ on the sky.

Repeated observations, each with an integration time of $120 \mathrm{~s}$, were made through a Johnson's $V$ filter, yielding a total number of 77 frames containing PKS 2316-423. The exposure time was fixed so that the counts at the central pixel of the blazar were about $25 \%$ below the saturation limit, well within the linear regime of the chip (nonlinear behavior only becomes significant for signals above $98 \%$ of the saturation level). A typical frame is shown in Figure 1, where we have marked the blazar position ("BL"), as well as the nearby galaxy MCG -07-47-032 ("G") located at

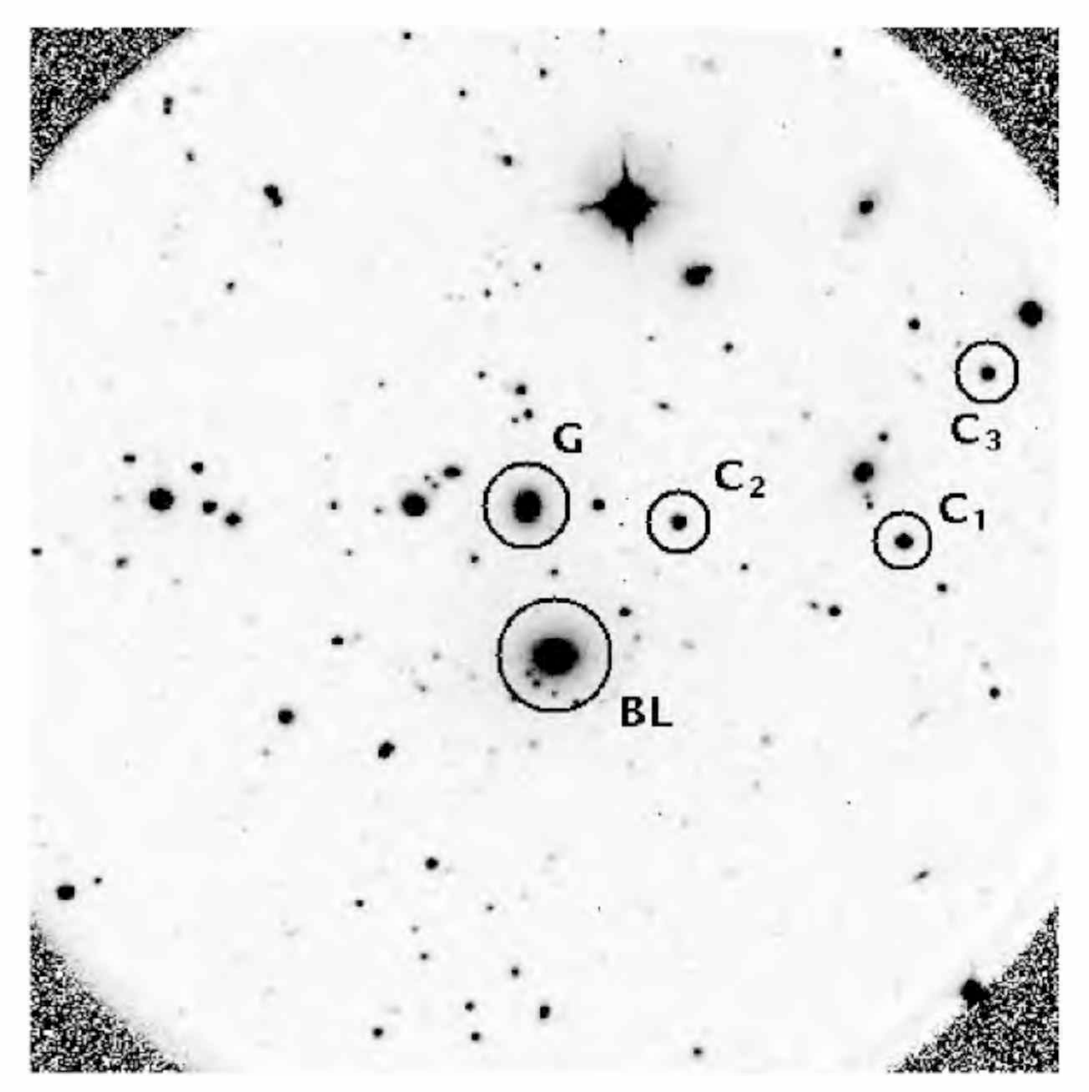

FIG. 1. - CCD frame showing the field of PKS 2316-423 (BL), with the galaxy MCG -07-47-032 (G) and starlike objects used for comparison and control purposes $\left(C_{1}\right.$ to $\left.C_{3}\right)$. The image is about $10^{\prime}$ on each side. North is up, and east to the left. 

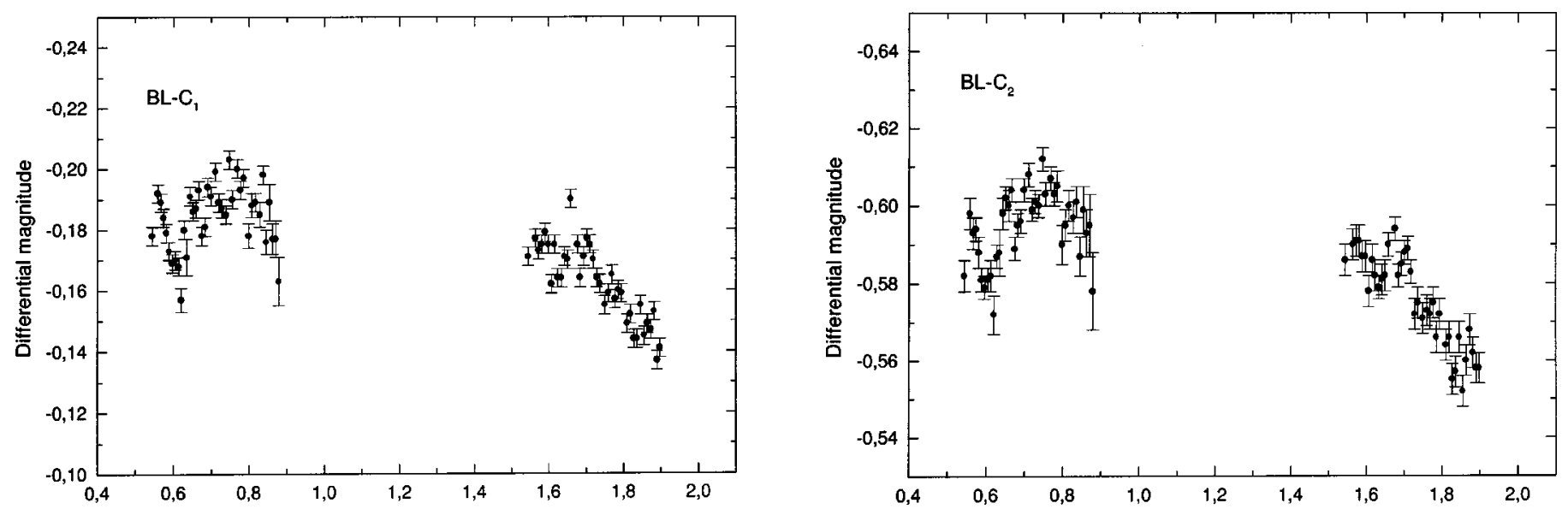

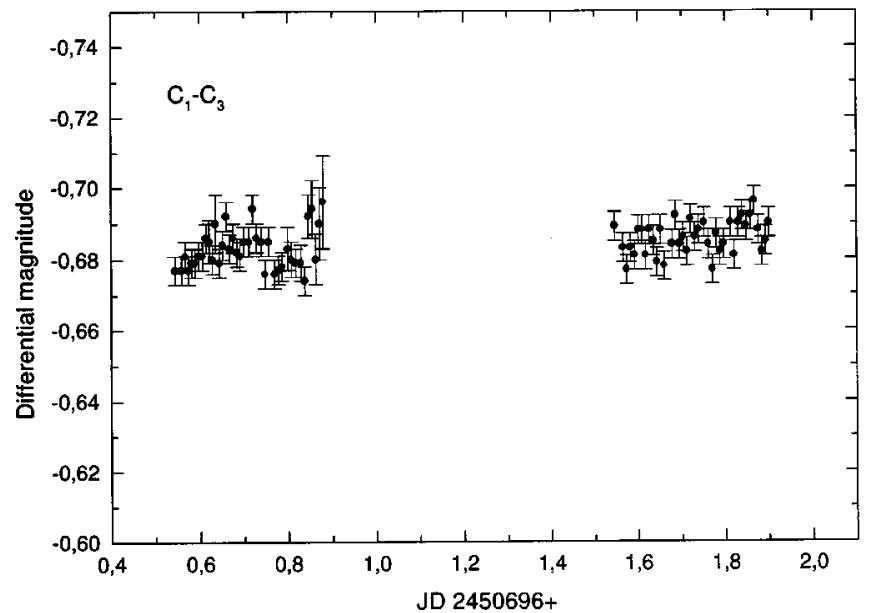

FIG. 2.-Differential light curve for PKS 2316-423 (top) and comparison star (bottom).

1.2 , and three stars (" $\mathrm{C}_{1}, "$ " $\mathrm{C}_{2}$," and " $\mathrm{C}_{3}$ ") used for control purposes. Calibration frames (bias and flat-field images) were taken each night before the observations.

The data reduction was made following standard procedures with the IRAF software package running on a Linux computer. All frames were debiased and flat-fielded using the normalized dome flats. Magnitude measurements were made relative to nonvariable field stars with the aperture photometry routine APPHOT, using an eight-pixel aperture, which is suitable for detecting changes in the AGN core but excludes galaxy contributions on photometric nights Romero et al. (1999).

Differential light curves were produced for the blazar minus a comparison star and the galaxy minus the same star, as well as for the comparison minus a control star. This

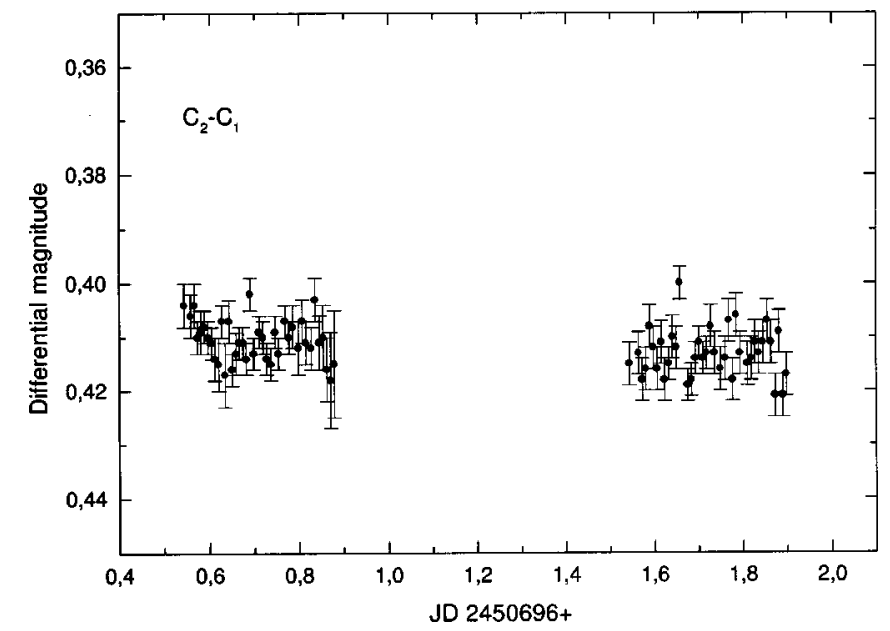

FIG. 3.-Same as Fig. 2, but with different comparison and control stars.

was made for different stars in order to allow multiple variability checking of the results (see Figs. 2, 3, 4, and 5).

\section{OBSERVATIONAL RESULTS}

A quantitative analysis of the results shown in Figures 2-5 can be seen in Table 1, where we list, for PKS $2316-423$ and the galaxy MCG $-07-47-032$, the different comparison and control stars used, the observational error $\sigma$ obtained from the standard deviation of the comparison light curve, the time spanned by the observations, whether the source classifies as variable according to the criterion described below, and the confidence level of the variability defined as $C=\sigma_{s} / \sigma$, with $\sigma_{s}$ the standard deviation of the source under study. We adopt here the variability criterion used by Jang \& Miller (1997) and other authors, which

TABLE 1

Microvariability Results WITH STARLIKe COMPARISONS AND CONTROLS

\begin{tabular}{ccccccc}
\hline \hline Object & Comparison & Control & $\sigma$ & $\Delta t$ & Variable? & $C$ \\
\hline PKS 2316-423........ & $C_{1}$ & $C_{3}$ & 0.0053 & $32.6 \mathrm{hs}$ & Yes & 2.99 \\
PKS 2316-423....... & $C_{2}$ & $C_{1}$ & 0.0043 & $32.6 \mathrm{hs}$ & Yes & 3.31 \\
MCG $-07-47-032 \ldots \ldots$. & $C_{1}$ & $C_{3}$ & 0.0053 & $32.6 \mathrm{hs}$ & Yes & 2.74 \\
MCG $-07-47-032 \ldots \ldots$ & $C_{2}$ & $C_{1}$ & 0.0043 & $32.6 \mathrm{hs}$ & Yes & 3.02 \\
\hline
\end{tabular}



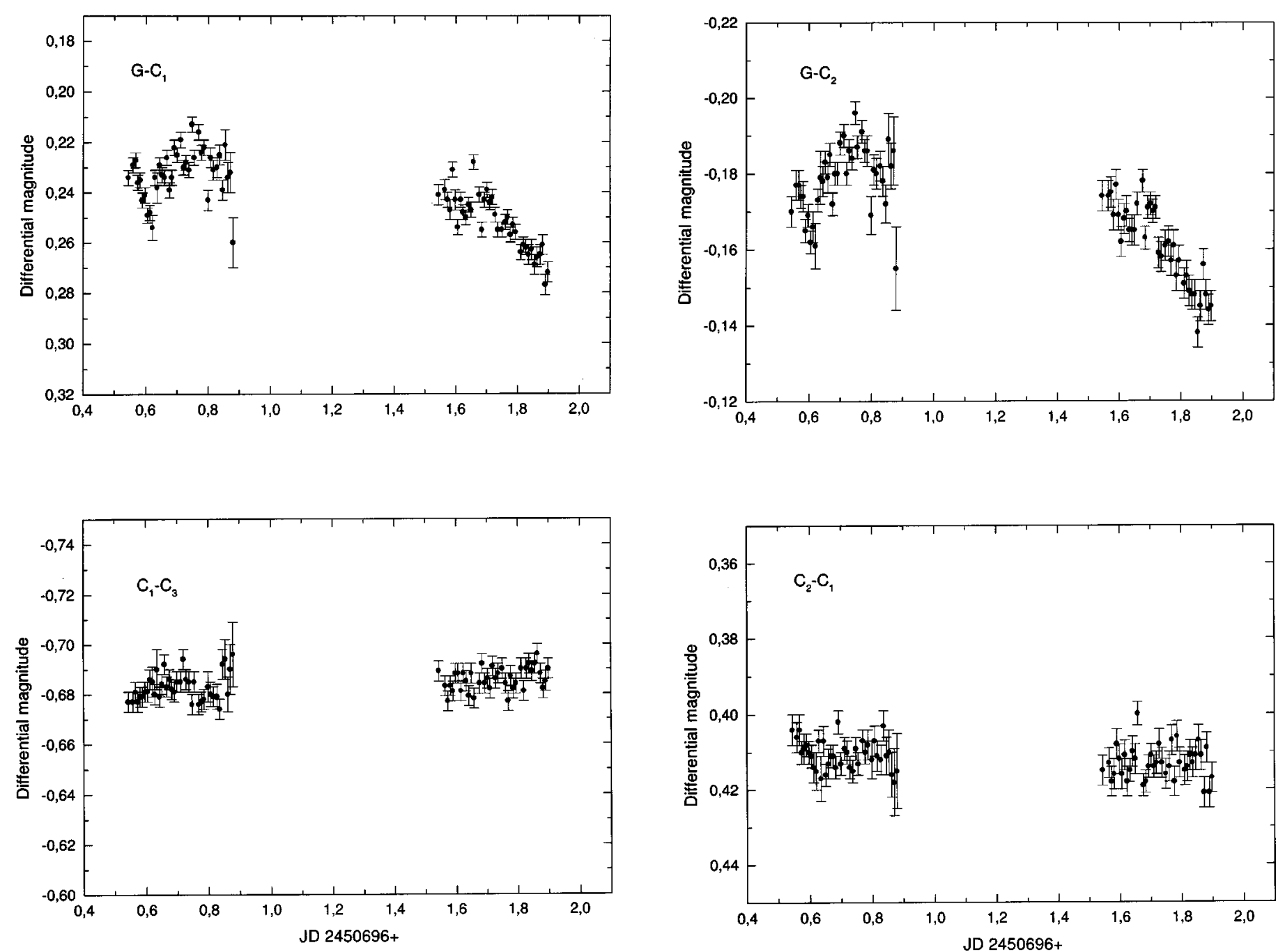

FIG. 4.-Same as Fig. 2, but for MCG -07-47-032

consists in considering a source variable if $C>2.6$, i.e., if the confidence is at a $99 \%$ level.

We see that PKS 2316-423 seems to be variable according to this criterion, being well above the fluctuations of the comparison light curves. Interestingly, the nearby galaxy presents almost exactly the same level of variations, with a similar light curve, also despite the fact that the comparison curve is well behaved and supposedly provides a confident control.

These facts strongly suggest that the observed variations are a spurious effect of the seeing fluctuations combined with the presence of a diffuse underlying component that is absent in the comparison and control stars. We can confirm this by making the differential light curve $\mathrm{BL}$ minus $\mathrm{G}$, as shown in Figure 6, and where there is no variability at all: the effects of seeing, which are not removed using star measurements, are banished when a similar extended object is used in the differential photometry.

In Figure 7 we show the FWHM temporal variability, which, not surprisingly, is very similar to the spurious light curves. The percentage fluctuation of the seeing is about $36 \%$, not very different from what is determined from PKS 2316-423 light curves. This trivial procedure of plotting the temporal variations in the FWHM constitutes a simple

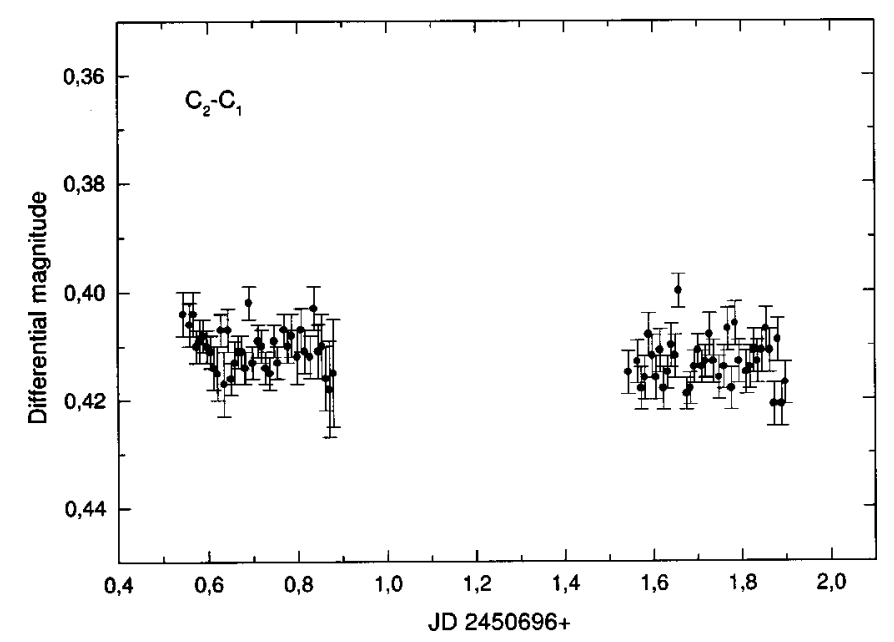

Fig. 5.-Same as Fig. 3, but for MCG -07-47-032

but powerful tool to discriminate whether the brightness variations are seeing induced or intrinsic to the sources.

It could be argued that the seeing conditions during the observing nights were too bad and that this effect is not important in most microvariability observations carried out

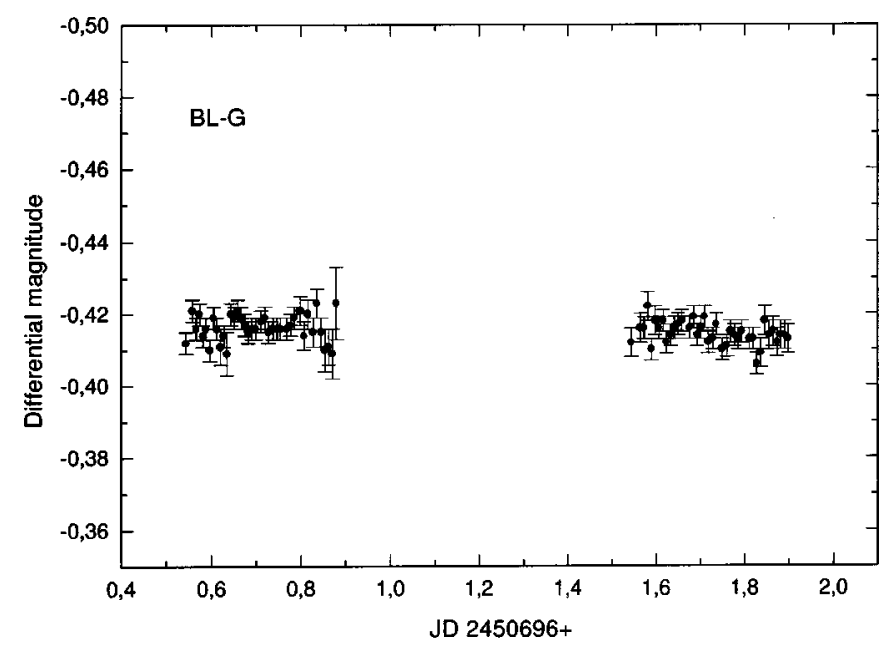

FIG. 6.-Differential light curve for PKS 2316-423 minus MCG $-07-47-032$. Notice that the spurious variability has been now removed. 


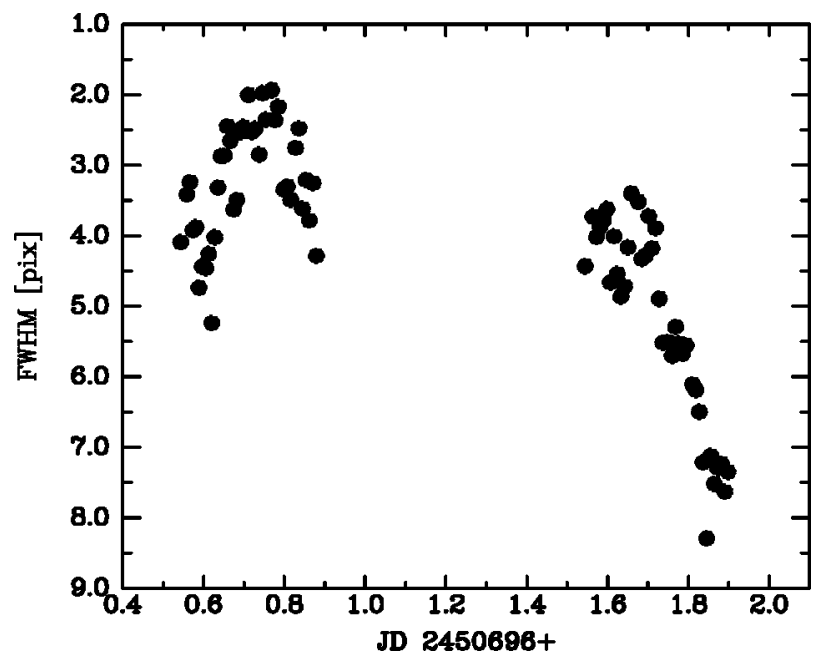

Fig. 7.-Atmospheric seeing evolution during the observations

under better weather. In order to ponder this we have made model simulations of the effect, which are described in the next section.

\section{SIMULATED OBSERVATION}

The surface brightness profile of the galaxy associated with PKS 2316-423 was measured from a co-added $V$ frame with an effective exposure time of $4560 \mathrm{~s}$. A de Vaucouleurs (1948) law produced a good fit, with an effective surface brightness $\mu_{e_{(V)}}=22.05 \mathrm{mag} \operatorname{arcsec}^{-1}$ and an effective radius $r_{e}=18.7$ (22.8 pixels). The resulting integrated magnitude is $V_{G}=13.7 \mathrm{mag}$, i.e., $0.4 \mathrm{mag}$ brighter than the value published by de Vaucouleurs et al. (1991). Subtraction of a model with these parameters yielded a central source with $V=16.8 \mathrm{mag}$, although this value should be taken with caution because of the uncertainties ( $\$ 0.2 \mathrm{mag}$ ) introduced by the subtraction procedure.

Taking the fit parameters as upper limits, both in surface brightness and size, a set of artificial images was generated simulating elliptical (i.e., following an $\boldsymbol{r}^{1 / 4}$ profile) galaxies with $25.05 \leq \mu_{e_{(V)}} \leq 22.05$, and $0.7 \leq r_{e} \leq 18^{\prime \prime} .7$, as if observed with our same instrumental setup and exposure times. Both round $(\epsilon=0)$ and flattened $(\epsilon=0.5)$ systems were considered. Two point sources were added to each frame, one at the center of the galaxy simulating a $V=16$ mag AGN and the other simulating a comparison star of the same magnitude at a projected distance of 210 pixels $\left(\sim 3^{\prime}\right)$, thus far enough so that the contribution from the galaxy was negligible at its location. Each frame was then convolved with two-dimensional (round) Gaussian functions of different FWHMs in order to simulate varying seeing conditions, from FWHM $=2$ to 8 pixels (i.e., between 1".6 and 6."6 with our scale, thus representing from normal to fairly bad conditions in many ground-based observatories). Finally, a constant sky value, photon noise, and read-out noise were added, matching our observing conditions and instrumental setup.

A similar set of artificial disk galaxies, following exponential laws with no bulge components, was also generated. Their central surface brightnesses ranged from $\mu_{0_{(\eta)}}=21.2$ to $\mu_{O_{(V)}}=23.9 \mathrm{mag} \operatorname{arcsec}^{-1}$, with scale lengths between $\alpha=12.3$ (15 pixels) and $\alpha=0$ ". 82 (one pixel). Thus, the brightest and largest model corresponds to a normal spiral $(\alpha \simeq 4.5 \mathrm{kpc}$; e.g., Kent 1985$)$ at $80 \mathrm{Mpc}$, while models with lower surface brightnesses and/or smaller sizes account for intrinsically fainter/smaller galaxies or normal galaxies at larger distances, affected by different amounts of extinction, cosmological dimming, etc. The same processing as for the elliptical models (point sources addition, Gaussian convolution, etc.) was applied to this set of images. Additional frames with two identical point sources were also generated and processed likewise to serve as control stars.

Aperture photometry of the simulated AGNs and comparison and control stars was done in a similar way as for the real observations, using a set of apertures with radii ranging from two pixels (1".6) to 20 pixels (16"4). Differential magnitudes $(\Delta m)$ were computed for each case, and the results are discussed in the following section.

\section{DISCUSSION}

Figure 8 shows $\Delta m$ (AGN minus comparison star) as a function of seeing FWHM for some selected models and different apertures. The integrated magnitudes of the model galaxies get dimmer from the top to the bottom rows; plots on a same row are represented with the same scale, so that elliptical and spiral models of similar integrated magnitudes can be directly compared.

Large variations in $\Delta m$ (up to $\sim 0.20 \mathrm{mag}$ ) against seeing FWHM are evident for the brightest models when the smallest apertures (two pixels, triangles; four pixels, crosses) are employed. Even for very faint galaxies $\left(V_{G} \simeq 19.5 \mathrm{mag}\right.$, i.e., approximately $3.5 \mathrm{mag}$ fainter than the simulated AGN; bottom row) $\Delta m$ varies up to about $0.02 \mathrm{mag}$ within the whole FWHM range. This is important, since such small apertures have been used previously in microvariability studies (e.g., Jang \& Miller 1997), following prescriptions made by Howell (1989). However, Howell's optimal dataextraction techniques, which require the use of aperture radii approximately matching the point-spread function FWHM, were explicitly developed for stellar (i.e., perfectly pointlike) sources, and we show here that meaningful errors can result when they are applied to an AGN embedded in a sufficiently bright host galaxy.

This problem is obviously circumvented by enlarging the photometric aperture. The same figure shows that variations are significantly smaller (although nonnegligible for bright hosts) when using large apertures (e.g., eight pixels, squares; or 20 pixels, circles). This effect is also observed when the aperture is increased for the analysis of the real data of PKS 2316-423. For this source, an aperture of 22 pixels results in a differential light curve with a confidence level of $C=1.14$, i.e., with almost the same dispersion as the comparison light curve. Unfortunately, the inclusion of a larger contribution from the host galaxy within the aperture is likely to dilute any intrinsic brightness variation of the AGN. Additionally, a degradation of the photometric quality arises from the use of larger apertures; for instance, apertures of 22 pixels result in errors that double those obtained with four pixels for PKS 2316-423. It is worth mentioning that the eight-pixel aperture corresponds to the largest FWHM measured during the observing run (see Fig. 7). Average values of about four pixels allow significant spurious variations in both observational and simulated light curves under strongly fluctuating seeing conditions.

Although elliptical and disk models of comparable magnitudes show similar behaviors, there is a hint that faint ellipticals are more affected by variations in seeing than comparably faint disk systems. However, this result could 

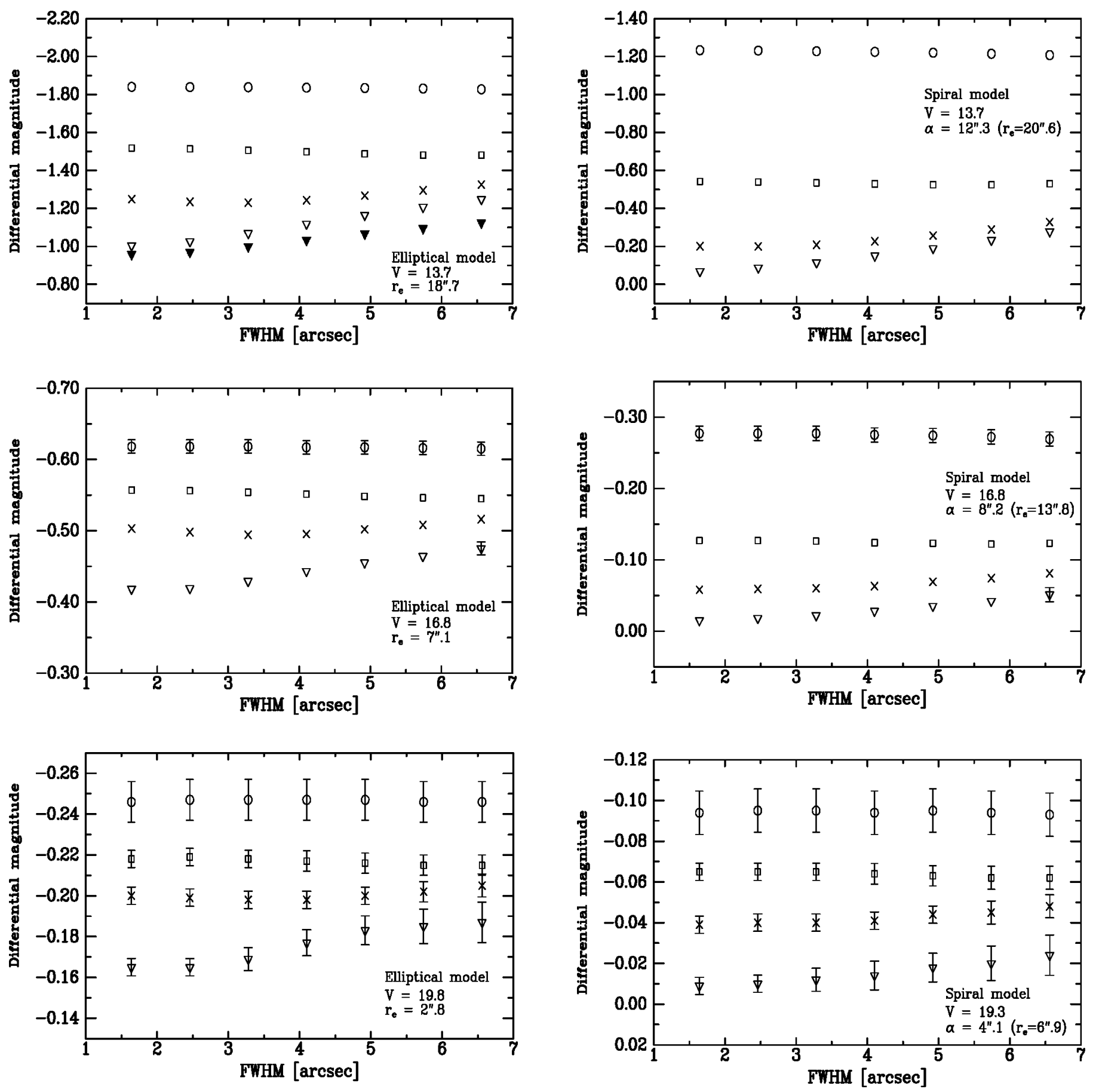

FIG. 8.-Differential magnitudes (AGN minus comparison star) as a function of PSF FWHM for several simulated host galaxies. Values obtained for round models using four different aperture radii are shown: two pixels (triangles), four pixels (crosses), eight pixels (squares), and 20 pixels (circles). Solid triangles correspond to flattened $(\epsilon=0.5)$ systems with a two-pixel aperture. Error bars are not shown when smaller than the symbols' size. An arbitrary vertical offset was applied to the data points in the two plots on the bottom row.

probably change with the inclusion of a bulge, not contemplated in our "spiral" models. Flattened models show qualitatively the same behavior as round ones; small differences are evident only with the smallest apertures, so we show just one such case in Figure 8 ( filled triangles in first panel).

Our data show that the maximum absolute variation in $\Delta m$ with seeing decreases both with decreasing effective radii and fainter surface brightnesses of the host galaxies. As a consequence, there is a strong correlation between the absolute variation in $\Delta m$ and the integrated magnitudes of the models. Figure 9 shows the amplitude of the variation in
$\Delta m$ averaged to $1^{\prime \prime}$ variation in seeing FWHM as a function of integrated magnitude $\left(V_{G}\right)$, both for elliptical (Fig. 9, left) and disk (or spiral) models (Fig. 9, right). Various aperture values are coded in the same way as for Figure 8.

It is clear, then, that using small apertures when measuring the magnitude of an AGN embedded in a host galaxy as bright or brighter than the AGN itself (recall that our simulated AGNs had $V=16 \mathrm{mag}$ in all cases), will result in spurious variations in $\Delta m$ amounting to several hundredths of a magnitude for seeing FWHM changes of $1^{\prime \prime}$ or more. However, noticeable variations are expected even using 

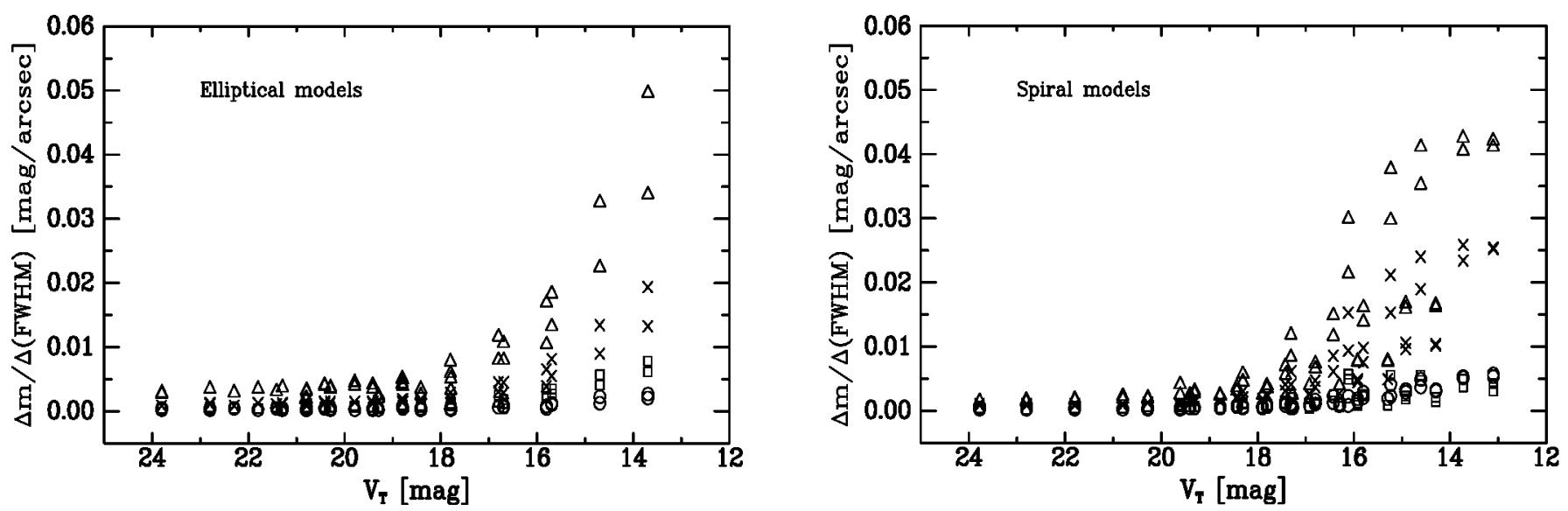

FIG. 9.-Amplitude of the variation in differential magnitude $(\Delta m)$ of simulated AGNs, averaged to $1^{\prime \prime}$ variation in seeing FWHM as a function of integrated magnitude $\left(V_{G}\right)$ of the host galaxy, for (left) elliptical models and (right) disk models. An AGN of $V=16$ mag was considered in all cases. The coding for the aperture values is the same as for Fig. 8.

fairly large apertures ( $\sim 6^{\prime \prime}$ in radius) for the brightest galaxies, like the host of PKS 2316-423. This is illustrated in Figure 10, which shows differential light curves for our brightest elliptical model and simulated comparison and control stars, using the actual seeing conditions of the night of 1997 September 4 and with aperture radii of two pixels (Fig. 10a) and eight pixels (Fig. 10b). Variability confidence levels for these curves are $C=31$ and $C=25$, respectively. Note that, for the larger aperture, $\Delta m$ is brighter when seeing is better, in very good qualitative agreement with our observations (see Figs. 2, 3, and 7); instead, the correlation is inverted for the two-pixel aperture. This can be explained as follows: within the small aperture the comparison star is relatively more affected by seeing than the shallower flux distribution of the galaxy plus starlike AGN; on the contrary, at the radius of the larger aperture the contribution from the star alone is small, but there is sufficient light from the galaxy so that this latter is more noticeably affected. When dealing with "real" data, observers might find it convenient to complement variability estimators such as formal confidence factors with additional criteria that take into account particular circumstances determined by the instrument characteristics, observing conditions, and other
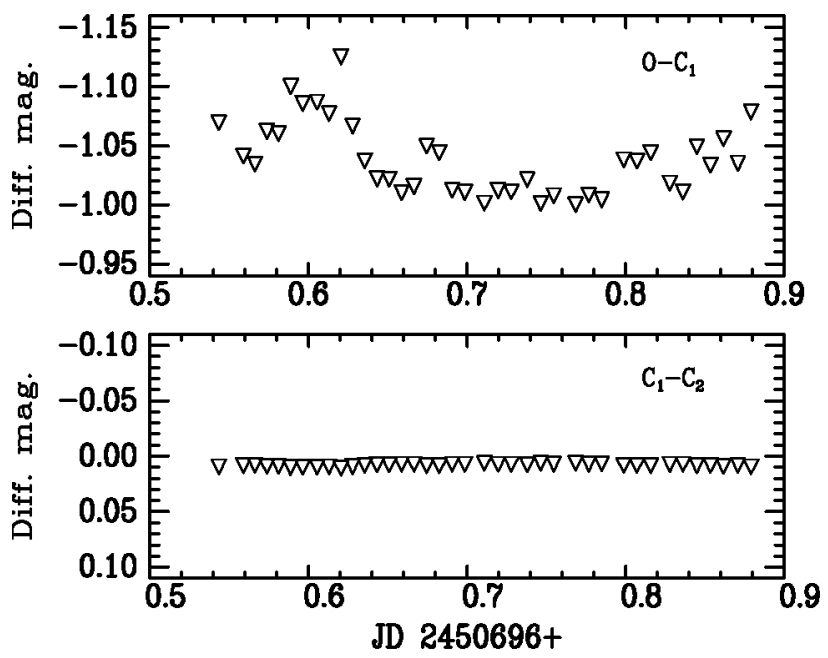

FIG. 10a factors occurring during the data acquisition/reduction process.

The fact that Carini et al. (1991) were able to remove all spurious magnitude variations due to seeing and/or transparency changes displayed by MCG 8-11-11 during their observations, does not necessary imply that the employed procedure results efficacious for any kind of objects observed under a wide variety of seeing conditions. MCG 8-11-11 is classified as a low surface brightness barred spiral (Malkan, Gorjian, \& Tam 1998 whereas most [although not all] quasar hosts turn out to be bright ellipticals Hutchings \& Neff 1992, Bahcall et al. 1997, Boyce et al. 1998); hence MCG 8-11-11 should not be considered as a representative example.

Summarizing, various factors contribute to determine the amount of the spurious variations in microvariability observations of quasars, like the shape, size, type and surface brightness profile of the host galaxy, and its magnitude relative to the AGN, as well as the particular conditions of the observations (seeing FWHM, sampling, etc.). Table 2 gives, for a $V=16 \mathrm{mag}$ AGN and various host galaxy magnitudes $V_{G}$, the minimum aperture radius that should be used to get no more than $0.01 \mathrm{mag}$ of spurious variation in $\Delta m$
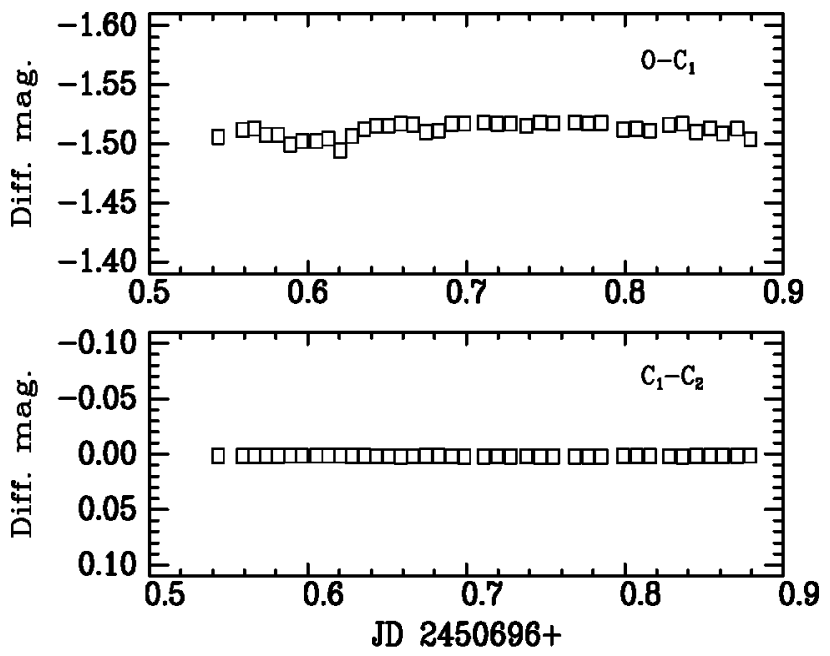

FIG. $10 b$

FIG. 10.- Simulated differential light curves: object minus comparison star $\left(O-C_{1}\right.$, top panels $)$ and control minus comparison star $\left(C_{1}-C_{2}\right.$, bottom panels), using (a) two-pixel (1".6) apertures and (b) eight-pixel (6".6) apertures. 
TABLE 2

Minimum APERTURe RAdi Recommended

\begin{tabular}{|c|c|c|}
\hline \multirow{2}{*}{$\begin{array}{c}V_{G} \\
(\mathrm{mag})\end{array}$} & \multicolumn{2}{|c|}{$R_{\mathrm{ap}}$} \\
\hline & (pixels) & (arcsec) \\
\hline $14 \ldots \ldots \ldots \ldots \ldots$ & 6 & 4.9 \\
\hline $15 \ldots \ldots \ldots \ldots \ldots$ & 5 & 4.1 \\
\hline $16 \ldots \ldots \ldots \ldots \ldots$ & 4 & 3.3 \\
\hline $17 \ldots \ldots \ldots \ldots \ldots$ & 3 & 2.5 \\
\hline $18 \ldots \ldots \ldots \ldots \ldots$ & 2 & 1.6 \\
\hline
\end{tabular}

for a $1^{\prime \prime}$ change in seeing FWHM, as it results from our simulations. A full treatment of the problem addressed in this paper would require more complete simulations, including the use of non-Gaussian PSFs and exploring the effects of crowded images, etc. Our results should thus be taken as a general starting point from which observers should critically evaluate their data according to their own instrumental setup and observational conditions.

\section{CONCLUSIONS}

We have shown observational evidence that the presence of a bright host galaxy may introduce spurious variations in microvariability observations of quasars. Our target, the BL Lac object PKS 2316-423, which is embedded within a bright elliptical galaxy, presented a differential light curve identical to that of a nearby galaxy within the same CCD frame, both closely following the seeing FWHM temporal fluctuations along the observing nights. It is worth noting that despite differential light curves between comparison and control stars showed no significant variations, meaning that all effects due to variable extinction and/or transparency were eliminated by the differential photometry technique, the effects of seeing on a bright host were still present.
Artificial images of AGNs within hosts spanning a broad range in magnitude were generated with different simulated seeing conditions. They were used to show that the amplitude of the spurious variations in differential magnitude caused by seeing fluctuations is correlated with host magnitude, and that it is higher for smaller photometric apertures. The analysis of our actual and simulated observations, then, allows us to make the following recommendations to future observers:

The photometric aperture should be carefully selected according to the magnitude of the host galaxy relative to the AGN itself, and the behavior of seeing along the observations. Very small apertures (with a radius similar to the PSF FWHM) should be avoided because variations of a few hundredths of a magnitude are possible, even with not very large $\left(\sim 1^{\prime \prime}\right)$ seeing fluctuations, and for faint hosts. These small variations, even if not sufficient to mimic a microvariability behavior, will at best increase the noise of the AGN light curve. On the other hand, large apertures will include a larger light contributions from the host galaxy, thus diluting any real AGN magnitude variation.

The case of AGNs within bright hosts should be treated with extreme caution, since small seeing fluctuations will produce large variations in $\Delta m$ for a broad range of aperture radii. The right choice of the aperture, consequently, should wisely weight all contributing factors in order to optimize the results.

This work has been supported by Fundación Antorchas and the Argentine Agencies CONICET (research grants PIP 0430/98 and PEI 0151/98) and ANPCT (PICT 0304881). The authors acknowledge use of the CCD and data acquisition system supported under U. S. National Science Foundation grant AST 90-15827 to R. M. Rich. They also thank valuable comments by the anonymous referee.

\section{REFERENCES}

Bahcall, J. N., Kirhakos, S., Saxe, D. H., \& Schneider, D. P. 1997, ApJ, 479, 642

Boyce, P. J., et al. 1998, MNRAS, 298, 121

Carini, M. T., Miller, H. R., \& Goodrich, B. D. 1990, AJ, 100, 347

Carini, M. T., Miller, H. R., Noble, J. C., \& Goodrich, B. D. 1992, AJ, 104, 15

Carini, M. T., Miller, H. R., Noble, J. C., \& Sadun, A. C. 1991, AJ, 101, 1196

Chakrabarti, S. K., \& Wiita, P. J. 1993, ApJ, 411, 602

Crawford, C. S., \& Fabian, A. C. 1994, MNRAS, 266, 669

de Vaucouleurs, G. 1948, Ann. d'Astrophys., 11, 247

de Vaucouleurs, G., de Vaucouleurs, A., Corwin Jr., H. G., Buta, R. J.,

Paturel, G., \& Fouque, P. 1991, Third Reference Catalogue of Bright

Galaxies (New York: Springer-Verlag) (RC3)

Gopal-Krishna, Sagar, R., \& Wiita, P. J. 1993a, MNRAS, 262, 963 1995, MNRAS, 274, 701

Gopal-Krishna, Wiita, P. J., \& Altieri, B. 1993b, A\&A, 271, 89

Howell, S. B. 1989, PASP, 101, 616

Howell, S. B., \& Jacoby, G. J. 1986, PASP, 98, 802

Hutchings, J. B., \& Neff, S. G. 1992, AJ, 104, 1

Jang, M., \& Miller, H. R. 1995, ApJ, 452, 582

. 1997, AJ, 114, 565

Kent, S. M. 1985, ApJS, 59, 115

Malkan, M. A., Gorjian, V., \& Tam, R. 1998, ApJS, 117, 25

Mangalam, A. V., \& Wiita, P. J. 1993, ApJ, 406, 420

Marscher, A. P., Gear, W. K., \& Travis, J. P. 1992, in Variability in Blazars, ed. E. Valtoaja \& M. Valtonen (Cambridge: Cambridge Univ. Press), 85

Miller, H. R., Carini, M. T., \& Goodrich, B. D. 1989, Nature, 337, 627

Miller, H. R., \& Noble, J. C. 1996, in ASP Conf. Series 110, Blazar Continuum Variability, ed. H. R. Miller, J. R. Webb, \& J. C. Noble (San Francisco: ASP), 17

Padovani, P., \& Giommi, P. 1995, MNRAS, 277, 1477

Rabbette, M., et al. 1998, A\&AS, 129, 445

Romero, G. E., Cellone, S. A., \& Combi, J. A. 1999, A\&AS, 135, 477

Sagar, R., Gopal-Krishna, \& Wiita, P. J. 1996, MNRAS, 281, 1267 